\title{
Local and global kinetic stability analysis of Alfvén eigenmodes in the 15MA ITER scenario
}

\author{
Ph. Lauber \\ Max-Planck-Institut für Plasmaphysik, EURATOM-Association, Garching, Germany \\ email: philipp.lauber@ipp.mpg.de
}

\begin{abstract}
The linear gyrokinetic spectral code LIGKA [1] is used to examine the Alfvén eigenmode stability properties of the ITER 15 MA scenario. It is shown that a kinetic multispecies treatment i.e. deuterium (D), tritium (T), helium ash (He), beryllium (Be), energetic $\alpha$ particles and neutral beam deuterium is indispensable for an accurate estimate of linear Alfvén mode properties. Comparisons to analytical expressions are carried out and several frequency ranges and modes such as the geodesic-acoustic/beta-induced Alfvén mode (GAM/BAE), the toroidal Alfvén eigenmode (TAE) and the ellipticity-induced Alfvén eigenmode (EAE) are discussed. A scan for the damping of TAEs (including radiative damping, continuum damping, ion and electron Landau damping) with the toroidal mode numbers $n=1-35$ is performed. Finally, the instability threshold as a function of the energetic particle $\beta$ is investigated for one example.
\end{abstract}




\section{Introduction}

The excitation of collective instabilities by super-thermal particles in hot plasmas and the related transport processes attract increasing interest due to their fundamental challenges for theoretical models and their practical importance for burning fusion plasmas. Within the last 10 years significant advances on both the theoretical and the experimental side have been made leading to a more detailed and quantitative understanding of fast-particle-driven instabilities. On the theoretical-numerical side, one of the crucial steps was to move from fluid models for the plasma background with a hybrid kinetic expression for the energetic particles (EPs) to a fully kinetic model for all the plasma species, i.e. background ions, background electrons, and fast ions[2, 1, 3, 4, 5, 6, 7, 8, 9, 10, 11, 12]. This improvement allows one to describe consistently the resonant interaction between global plasma waves such as shear Alfvén and Alfvén-acoustic waves, and the particles via Landau damping, i.e. the dynamics parallel to the magnetic background field. The accurate treatment of the plasma background leads not only to changes in the linear mode properties such as frequency, growth/damping rate, and mode structure but also influences the non-linear dynamics.

Due to major advances on the diagnostics side in present day experiments, the comparison of these advanced models with the experiments can be carried out in a more detailed and comprehensive way than a few years ago. For example, the measurement of damping rates via active external antennas [13, 14], the imaging of 2D mode structures via electron-cyclotronemission spectroscopy [15, 16, 17, 18], or the direct detection of escaping fast ions [19] allows one to diagnose various kinetic features of the plasma modes that are responsible for the transport of EPs. Furthermore, the fast particle distribution function itself can also be measured with much greater confidence [20, 21]. Therefore, the new physics accessible due to a more comprehensive model and numerical implementation can be directly verified and validated with experimental data. Based on this validation on present day experiments, predictions for ITER in several scenarios can be attempted, which is the topic of this paper. Previously, an analysis has been carried out in ref. [22] using the hybrid kinetic code NOVA$\mathrm{K}$ [23]. In this approach the eigenmode structure was determined by the ideal code NOVA and the damping and drive were calculated perturbatively. In ref.[24] local linear gyrokinetic estimates for the fusion alpha density profile in ITER were given. In contrast, the treatment in this paper is global, kinetic and non-perturbative. The reasons for relaxing the nonperturbative and non-local constraints are the following: first, finite Larmor radius effects change the mode structures by coupling to the kinetic Alfvén wave via radiative damping [25] and via direct interaction with the continuum. The emerging short wave length features and the small real frequency shifts (continuum intersection!) are important for correctly describing the damping of these waves. Secondly, it will be shown in this paper that not always the 'main' TAE gap modes with $(m, m+1)$ as dominant poloidal harmonics are the least damped TAEs for a given toroidal mode number $n$. There is evidence [26] that these branches (as described in section 3.3) can become dominant in the non-linear phase.

After briefly describing the LIGKA code [1] in section 2, a local kinetic analysis of the 15 MA standard scenario (profiles in section 3.1) is given. Several models of complexity are 
compared, dilution effects due to helium ash ( $\mathrm{He})$ and beryllium $(\mathrm{Be})$ described and a large range of mode numbers and frequencies are investigated. Other impurities such as tungsten are not included, since their contribution to the physics of Alfvén modes are negligible: transport studies show that in order to reach $Q>5$ the tungsten concentration must be below $0.007 \%$ [27]. This small amount has no effect on either the Alfvén velocity or on a reduced ion Landau damping, even when high charge states are considered. Section 3.3 shows the results of global mode calculations and its destabilisation due to alpha particles and beams (section 3.4). Due to the sensitivity of global mode stability on the profiles for the background quantities and the EP distribution functions, no complete analysis for this scenario can be given here, however, a first set of representative parameters is investigated, also in the view of code-code benchmarking. A discussion on the profile sensitivity and on other consequences from this linear analysis will be given in the conclusion section.

\section{Theoretical model}

The equations solved by LIGKA are the quasi-neutrality equation (QN) and the gyrokinetic moment equation (GKM) that together with the gyrokinetic equation for the particle distribution functions form a consistent model for electromagnetic perturbations in tokamak geometry [2, 3]. Starting directly from the implemented equations, a rather general dispersion relation can be derived ([28] eqn.12,[29] eqn.4,[3, 11]), allowing the analysis of the continuum solutions and the local damping due to electron and ion Landau damping, here written for multiple ion species:

$$
\begin{gathered}
\frac{\omega^{2}}{\omega_{A}^{2}}\left(1-\frac{\omega_{p}^{*}}{\omega}\right)-\bar{k}_{\| m}^{2} R_{0}^{2}=\sum_{a=i, e} 2 \frac{v_{t h, a}^{2}}{\omega_{A}^{2} R_{0}^{2}}\left(-\left[H\left(x_{a, m-1}\right)+H\left(x_{a, m+1}\right)\right]+\right. \\
\left.\tau_{a}\left[\frac{N^{m}\left(x_{a, m-1}\right) N^{m-1}\left(x_{a, m-1}\right)}{D\left(x_{a, m-1}\right)}+\frac{N^{m}\left(x_{a, m+1}\right) N^{m+1}\left(x_{a, m+1}\right)}{D\left(x_{a, m+1}\right)}\right]\right)
\end{gathered}
$$

where $\bar{k}_{\| m}^{2}$ stands for the parallel wave vector including the toroidal coupling up to first order

in $\epsilon$, e.g. $\bar{k}_{\| m}^{2}=\left(k_{\| m}^{2}+k_{\| m+1}^{2} \pm \sqrt{\left(k_{\| m}^{2}-k_{\| m+1}^{2}\right)^{2}+4 \hat{\epsilon}^{2} r^{2} k_{\| m}^{2} k_{\| m+1}^{2}}\right) /\left(2\left(1-\hat{\epsilon}^{2} r^{2}\right)\right)$ where $\hat{\epsilon}$ is $\hat{\epsilon}=5 r / 2 R_{0}[30,31]$. Further,

$$
x_{a, m}=\frac{\omega}{\left|k_{\|, m}\right| v_{t h, a}} ; \quad v_{t h, a}=\sqrt{\frac{2 T_{a}}{m_{a}}} ; \quad \omega_{p}^{*}=\sum_{i} \frac{k_{\theta}}{e B n_{i}} \frac{\partial p_{i}}{\partial r} \frac{m_{i} n_{i}}{m_{e f f} n_{e}} \quad \tau_{a}=T_{e} / T_{a} .
$$

The definitions for the polynomials $H, N, D$ including the complex plasma dispersion function can be found e.g. in the references [28, 11]. The upper index $m$ in $N^{m}\left(x_{m-1}\right)$ refers to the poloidal mode number $m$ to be used in $\omega_{m}^{*}=\frac{T_{i}}{e B} k_{m, \theta} \frac{\nabla n}{n}$.

This very general dispersion relation describes the linear physics of shear Alfvén waves in tokamak geometry (left hand side) and their modification due to the coupling to acoustic and kinetic ballooning waves up to first order in $\epsilon$ (right hand side). Note that both electron and ion Landau damping are included.

Since this dispersion relation can be derived directly from the equations that are the basis for LIGKA, all simplifications concerning the geometry and $v_{\perp}\left(v_{\perp}=0\right)$ that are assumed 
Local and global kinetic stability analysis of Alfvén eigenmodes in the 15MA ITER scenario4
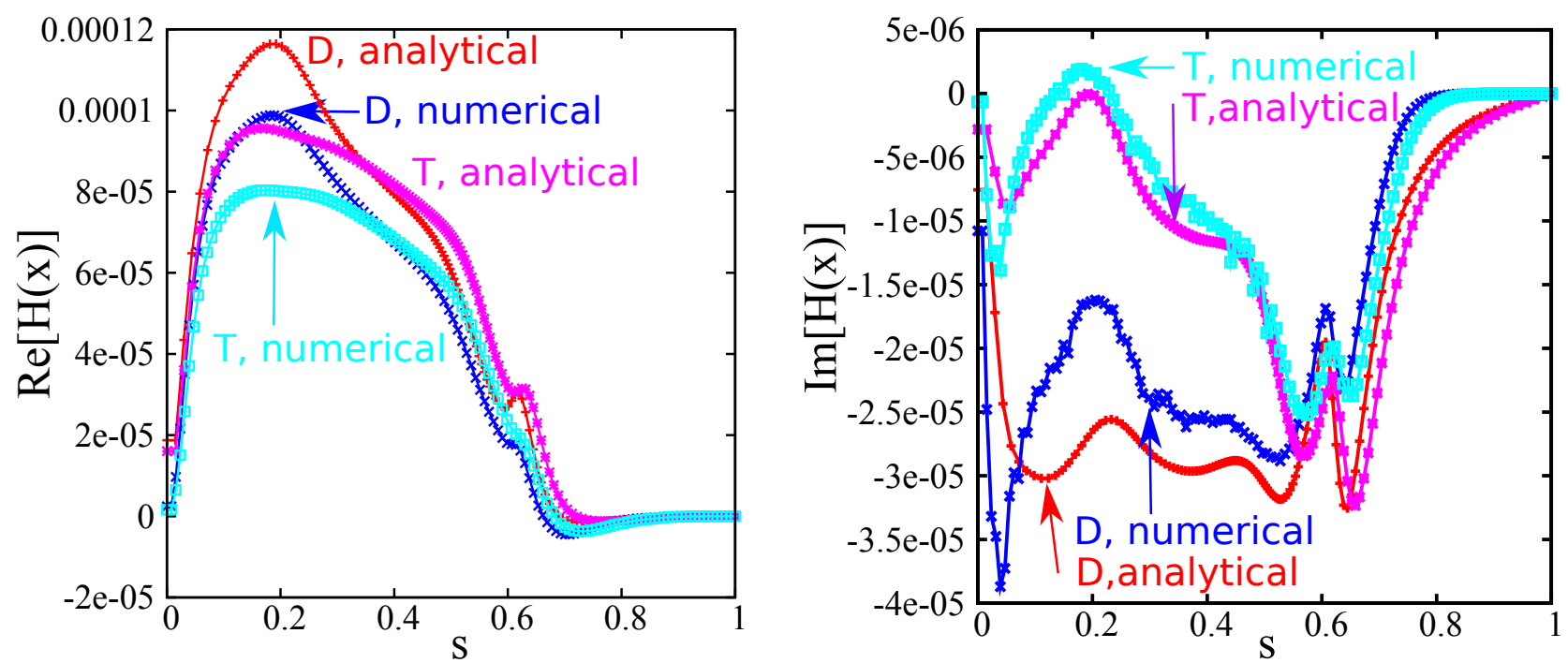

Figure 1. comparison of the analytical and the numerical value of the real part (left) and the imaginary part (right) of the coefficient $H(x)$ as given in eqn. 1 for $\mathrm{D}$ and $\mathrm{T} ; \omega$ was chosen to be close to the lower TAE accumulation point $(f=71.2 \mathrm{kHz})$ with a damping of $2.5 \%$.

when expanding the drift operator can be dropped if the coefficients and the matrix elements of the GKM and the QN equations are calculated numerically [11]. Although the influence of the elongation of flux surfaces [32] and deeply trapped particles [33] on the dispersion relation have been analytically examined, in general it is required to numerically evaluate the coefficients in eqn. 11. The LIGKA code has been benchmarked [34, 11] to recover this analytical dispersion relation in the appropriate limit. Figure 1 shows an example for the differences between the numerical and the analytical evaluation of one of the relevant coefficients $H(x)$. Unless stated otherwise, the coefficients are evaluated numerically for the local and global analyses in sections 3.2 and 3.3 .

As indicated by the definition of $\omega_{*}$ and the sum on the right hand side of eqn. 1 , in this version of the dispersion relation the thermal ion species are treated separately, i.e. their orbit and profile properties are taken into account explicitly in order to account for D-T mixture and dilution effects due to $\mathrm{He}$ ash and Be impurities.

According to this and the previous section, the Alfvén velocity is defined as

$$
v_{A}=\frac{B}{\sqrt{\mu_{0} \sum m_{i} n_{i}}} .
$$

This results in an on-axis value for $f_{A}=v_{A} /\left(2 \pi R_{0}\right)$ of $178 \mathrm{kHz}$ for a 50:50 D-T mixture with the given (figure 2) $\mathrm{He}$ ash and Be concentration.

The profiles given in figure 2 are based on on ASTRA transport simulations for the ITER baseline scenario with a plasma current $I_{p}=15 \mathrm{MA}, R_{0}=6.2 \mathrm{~m}, a=2 \mathrm{~m}$ and a magnetic field of $B=5.3 \mathrm{~T}$ [35]. Two NBI beam sources with $16.5 \mathrm{MW}$ each are considered (one onaxis and one off-axis) and the fusion gain $Q$ is 10 for these parameters. The on-axis safety factor is chosen to be $q_{0}=0.99$ unless stated otherwise. The toroidal $\beta$ is $2.8 \%$ and the on-axis thermal and $\alpha$-particle $\beta$ s are $6.84 \%$ and $1.15 \%$, respectively. 

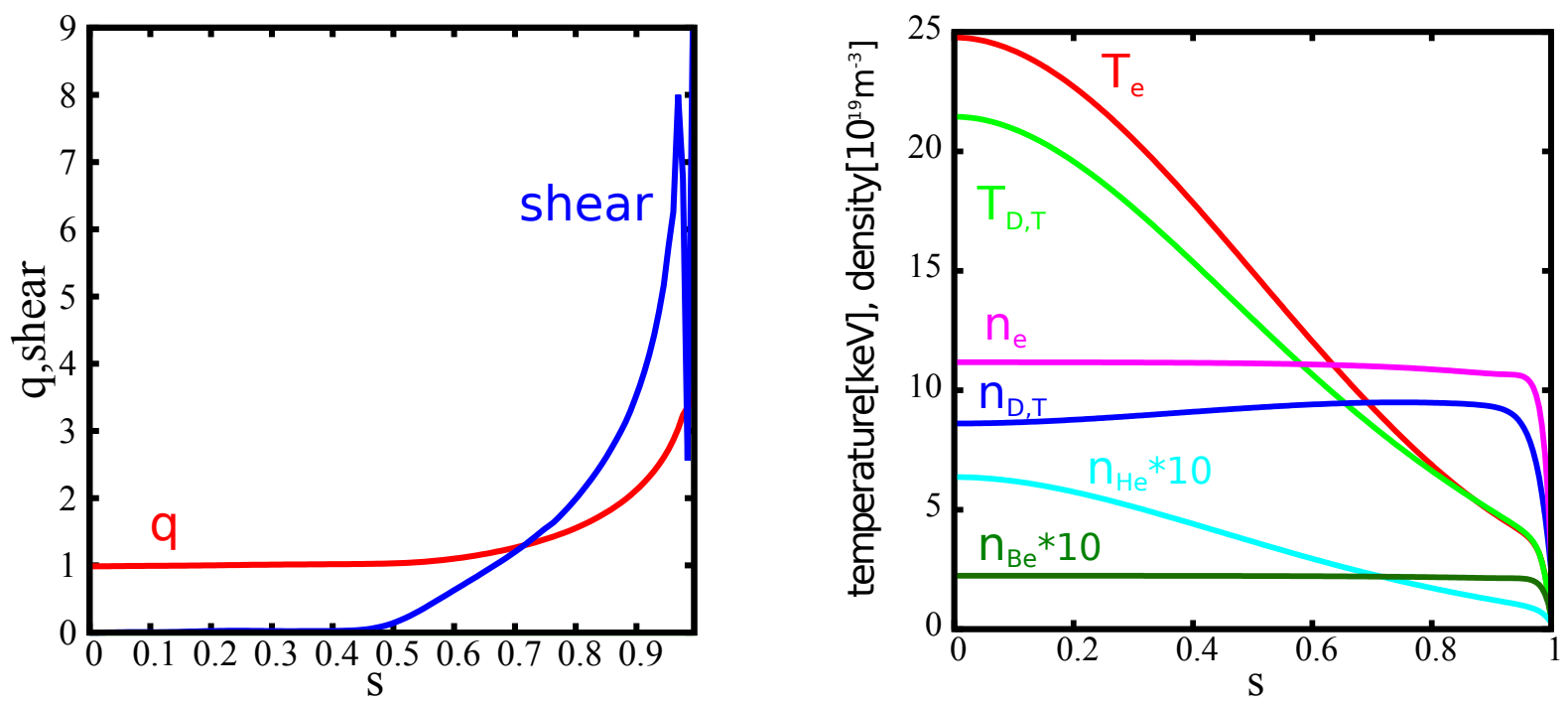

Figure 2. Left: $q$ and shear profile used in the simulations; Right: temperature and density profiles for the background species: the tritium and the impurity ions are assumed to have the same temperature as the deuterium and the tritium. Note that the densities of the $\mathrm{He}$ ash and the $\mathrm{Be}$ are multiplied by 10 in this plot.
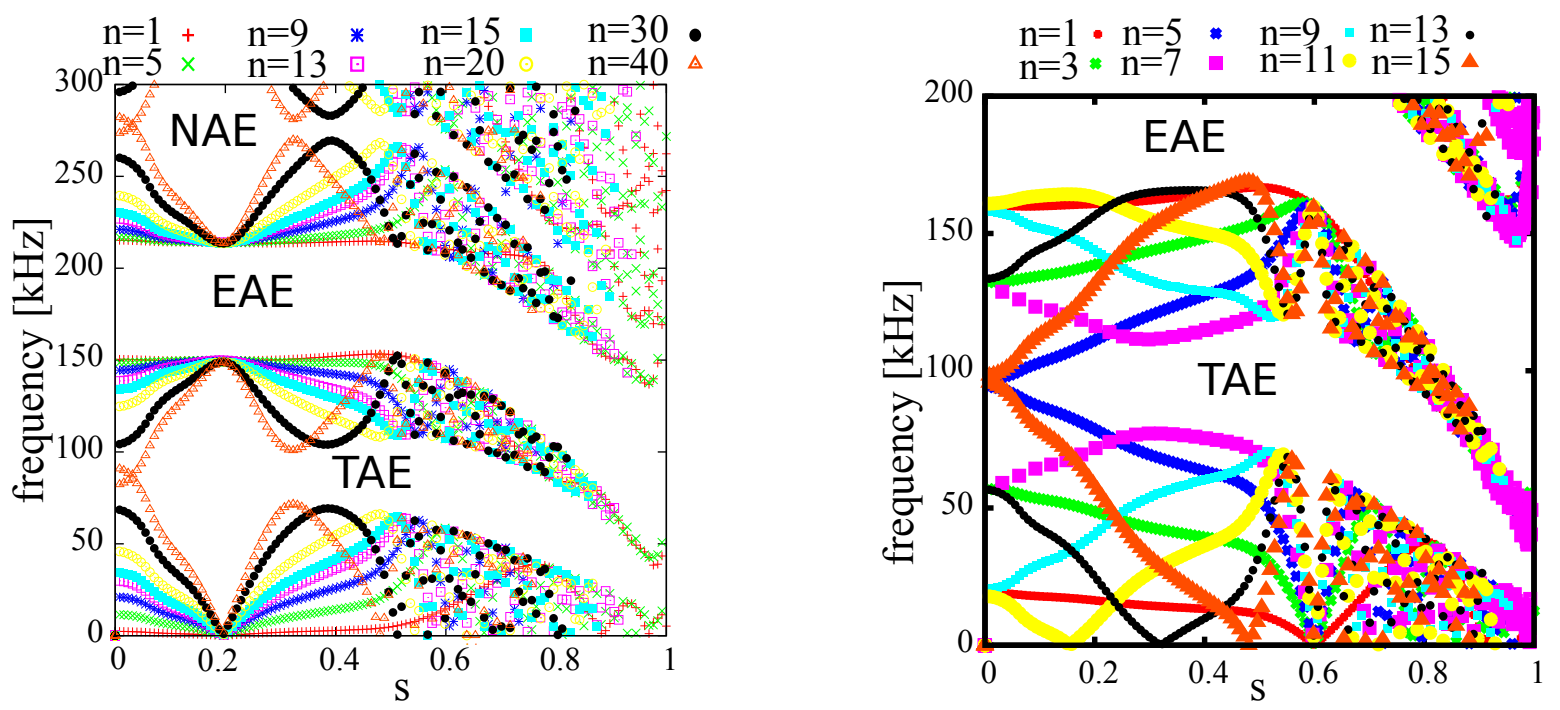

Figure 3. Left: ideal shear Alfvén wave continuum (SAW) for $q_{0}=0.99$. The gap around 80 $\mathrm{kHz}$ is the TAE (toroidicity induced) gap, the gap at $180 \mathrm{kHz}$ is the EAE (ellipticity induced) gap, and the gap at $\sim 275 \mathrm{kHz}$ is the NAE (non-circular triangularity-induced) gap. Right: ideal SAW continuum for $q_{0}=0.9$; core localised gaps for low and medium $n$, here e.g. for $n=7$ can exist.

\section{Numerical results}

\subsection{Ideal MHD spectra and TAE location}

As a start, in figure 3 the ideal MHD shear Alfvén spectra (SAW) for different $n$ are plotted for the $q$-profile with $q_{0}=0.99$ as given in figure 2. The TAE gap for low- $n$ TAEs is outside $s \geq 0.45$ due to the vicinity of $q$ to 1 . Here $s$ is the square root of the normalised poloidal 
flux. The gaps are closed, for the even TAEs at the bottom of the gap at around $s \sim 0.85$ and for the odd TAEs at $s \sim 0.6$. Carrying out a sensitivity study with respect to $q_{0}$ [36] shows that the gap remains closed for reasonable choices for $q_{0}$ between $0.9<q_{0}<1.15$.

Due to the flatness of the $q$-profile and the fact that $q$ is very close to 1 , the radial TAE position $q_{T A E}=\frac{m+1 / 2}{n}$ for increasing mode number moves monotonically from $s \sim 0.7$ to $s \sim 0.35$. This means that the modes form radially a dense cluster that motivates the question regarding the extent to which non-linear mode saturation effects (resonance overlap) alter quasi-linear predictions. In contrast to that, for $q_{0}=0.9$, core localised gaps with TAEs that are also sometimes called 'tornado modes' [37, 38, 39] can exist. Due to formation of this 'inner' gap at $s<0.4$, the main TAE gap can be closed in the centre (see figure 3, right). The details of the case with $q_{0}=0.9$ are analysed in ref.[36].

\subsection{Local kinetic analysis}

The kinetic dispersion relation eqn. 1] was used to benchmark the coefficients entering the global solver of LIGKA. As shown previously [11], in the appropriate limit (e.g. electrons in a circular equilibrium) the analytical expressions can be recovered numerically, also in the case where the imaginary part of $\omega$ is negative. Mesh refinement close to the resonances and rational interpolation techniques for determining the residual are employed. Figure 1 shows this comparison for the ITER case described in the previous section. The first observation is that indeed analytical theory and numerical evaluation agree relatively well, however, the damping is over-estimated by the analytical expression (eqn. 1). This is expected because of the assumption of well circulating ions $\left(v=v_{\|}\right)$in the analytical derivation [11]. Secondly, there is a large difference for $\mathrm{D}$ and $\mathrm{T}$, that is especially important for the imaginary (damping) part: due to their larger mass $\mathrm{T}$ ions contribute much less to the total ion Landau damping than D. It is emphasised that the damping depends exponentially $\sim e^{-x^{2}}$ (see eqn 1 ) on the argument $x_{m}=\frac{\omega}{\left|k_{\|, m}\right| v_{t h}}$. This demonstrates the importance of a fully kinetic treatment: the ion Landau damping is very sensitive on the mode frequency, local temperature, the local aspect ratio and the isotope mix.

Electron Landau damping is rather small due to the large ratio of $v_{t h, e} / v_{A} \sim 13$ (on -axis). Only very close to rational surfaces $\left(k_{\|}=0\right)$ small 'spikes' in the coefficients $H(x), N(x)$ and $D(x)$ can make a small contribution (not shown here). Compared to the ideal, reduced MHD results in figure 3, considerable corrections occur due to the coupling to the sound waves and drift waves, especially for higher mode numbers. These corrections cannot be captured properly by one single compressibility constant since the term in large round brackets on the right hand side of eqn.1 depends on various quantities, most importantly on the frequency as an argument of the plasma dispersion function. Figure 4 (left) shows this comparison between the reduced MHD calculation and the kinetic continuum for $n=20$ that was chosen because it is an 'intermediate' case between local $(n>20)$ and global $(n<20)$ mode structures (see also figure 8). In figure 6 (left) the local damping for the kinetic spectrum is shown: as expected for higher frequencies, the ion Landau damping is reduced ( $\operatorname{smaller} x$ ). At lower 

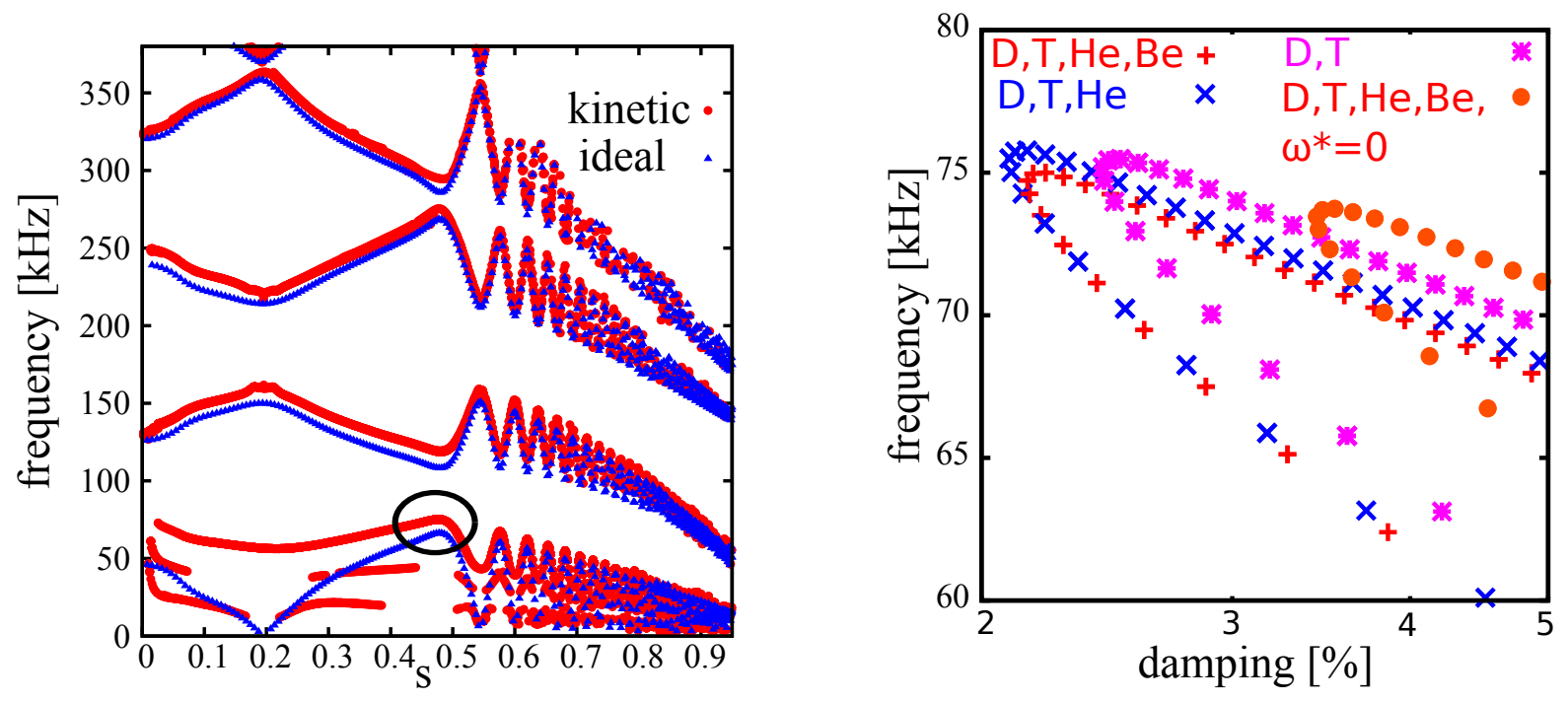

Figure 4. Left: kinetic continuum for $n=20\left(q_{0}=0.99\right)$. In red, the kinetic calculation, in blue the ideal spectrum. The black circle indicates the part of the spectrum that is shown on the right.; Right: the lower TAE continuum branches as on the left as a function of the local damping for different isotope mixes: the red crosses refer to a mix with D:T 50:50, He ash and $2 \%$ (of the electron density) Be. The blue diagonal crosses include only $\mathrm{D}, \mathrm{T}$ and $\mathrm{He}$ ash, and the magenta stars only D and T. The red dots show the damping when all $\omega_{*}$ effects are neglected (D,T,He ash,Be).

temperatures, i.e. larger minor radius the damping decreases. Therefore, the local damping is much smaller for the odd (top of the TAE gap) TAE branch since it has a higher frequency than the even (bottom of the gap) TAE. However, a global analysis shows that the continuum damping for odd modes is stronger since the gap is closer to the continuum formed by the higher poloidal mode branches.

On the right of figure 4, the local (numerical) solution of the dispersion relation for the even TAE ( $n=20$ ) branch is plotted. As discussed above, the background plasma composition is shown to be crucial for the correct estimation of the damping: especially adding the He ash (difference between pink stars and blue diagonal crosses) decreases the damping considerably at the accumulation point (maximum of the real frequency). Since for $n=20, m=20 / 21$, $\omega_{*}$ is rather large due to a large $k_{\theta} \sim-m / r$, the diamagnetic effects make a rather large impact on the local damping rate. It should be noted that in all cases with less than 4 species the densities were slightly adapted in order to fulfill (numerically) quasi-neutrality. Also the differences due to changed Alfvén velocities via the mass density were taken into account. The variation of the ratio of $\mathrm{D}$ and $\mathrm{T}$ around the optimal ratio $1: 1$ is not causing large differences in the damping [36].

There is a considerable difference between taking into account circulating particles only and both circulating and trapped particles. Due to their smaller bounce frequency trapped particles interact less efficiently with TAEs. A reduced damping (1.8\% vs. $2.2 \%)$ is the consequence (see figure 5, left). This effect is more pronounced when the stability and the excitation of low-frequency branches (BAE, AITG,KBM,...) are investigated [34]. 
Local and global kinetic stability analysis of Alfvén eigenmodes in the 15MA ITER scenario8
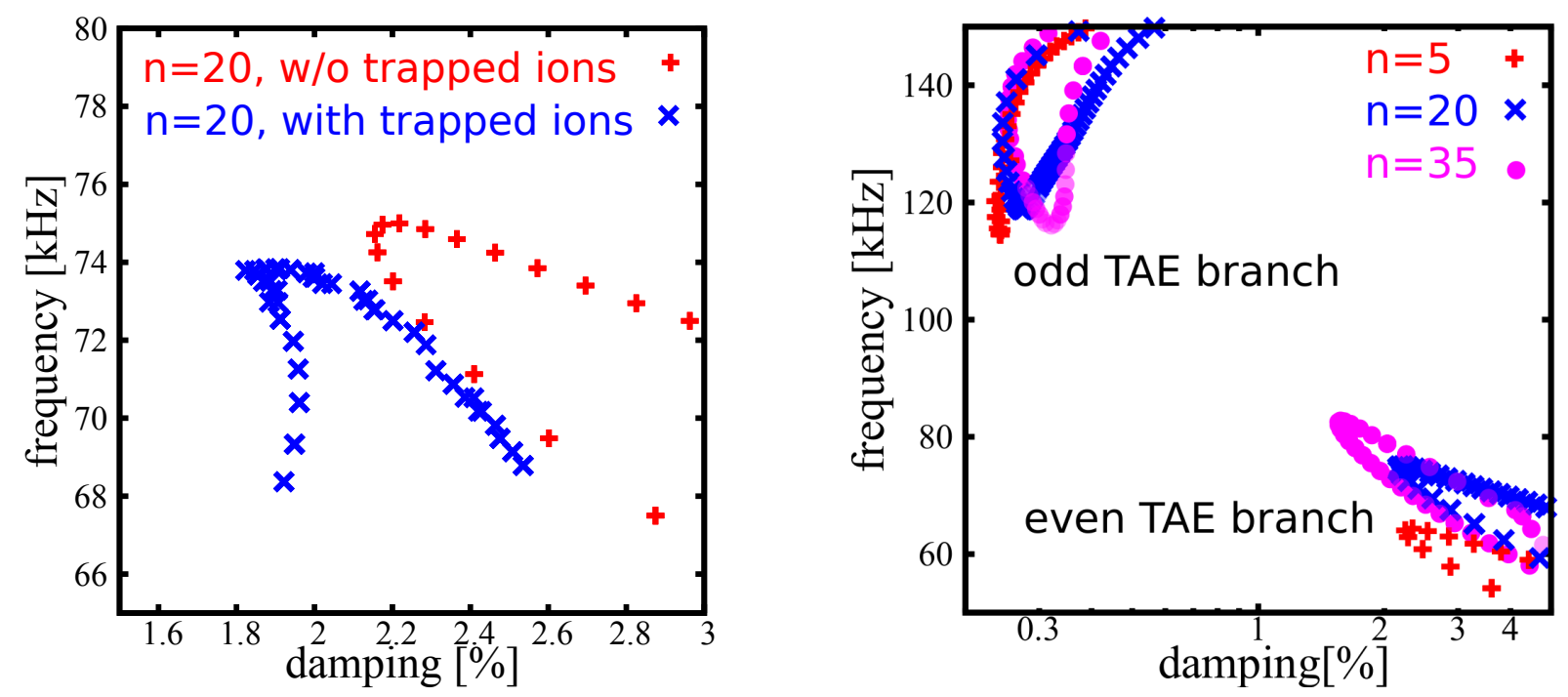

Figure 5. Left: kinetic continuum for $n=20$ for circulating particles only (red crosses) and circulating and trapped particles (blue diagonal crosses); Right: kinetic continuum for the TAE branch for different toroidal mode numbers $n$;
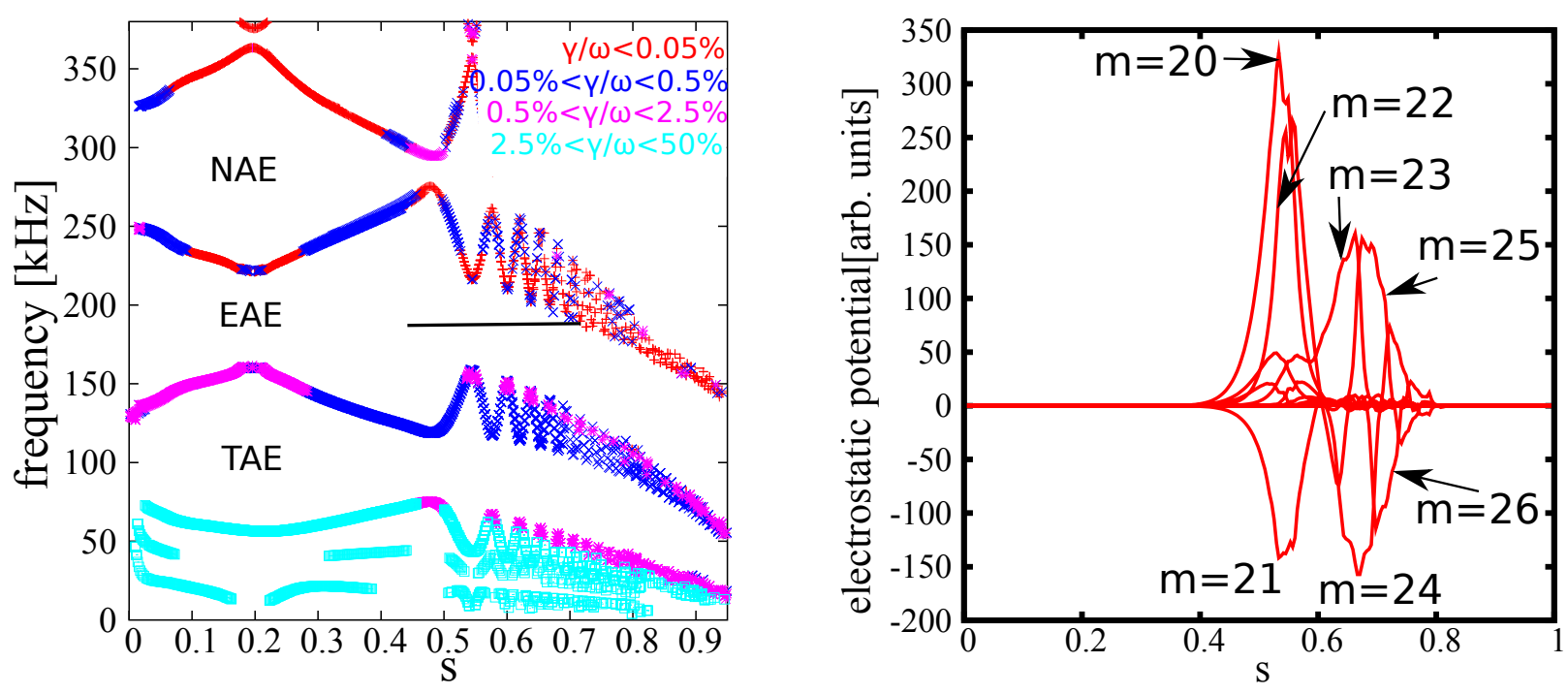

Figure 6. Left: kinetic continuum for $n=20$ in the EAE frequency range: the red crosses show the part of the continuum that has a damping less than $\gamma / \omega<0.05 \%$, the blue diagonal crosses $0.05 \%<\gamma / \omega<0.5 \%$, the magenta stars $0.5 \%<\gamma / \omega<2.5 \%$, and the cyan squares $2.5 \%<\gamma / \omega<50 \%$; Right: $n=20$ EAE mode with a frequency of $190 \mathrm{kHz}$ and a damping rate of $\gamma / \omega=0.6 \%$; the mode frequency and its width are marked with the black line in the spectrum on the left.

In figure 5 (right) the damping characteristics for the even (lower frequency) TAE branch are plotted for some selected toroidal mode numbers. Although the TAE gap moves radially inwards in regions with higher ion background temperature, the damping decreases for high mode numbers. Two effects are responsible for this: the frequency increases due to $\omega_{T A E} / \omega_{A 0}=\frac{n}{2 m+1}$ from $0.33 \omega_{A 0}$ towards $0.5 \omega_{A 0}$ and the diamagnetic effects increase for 

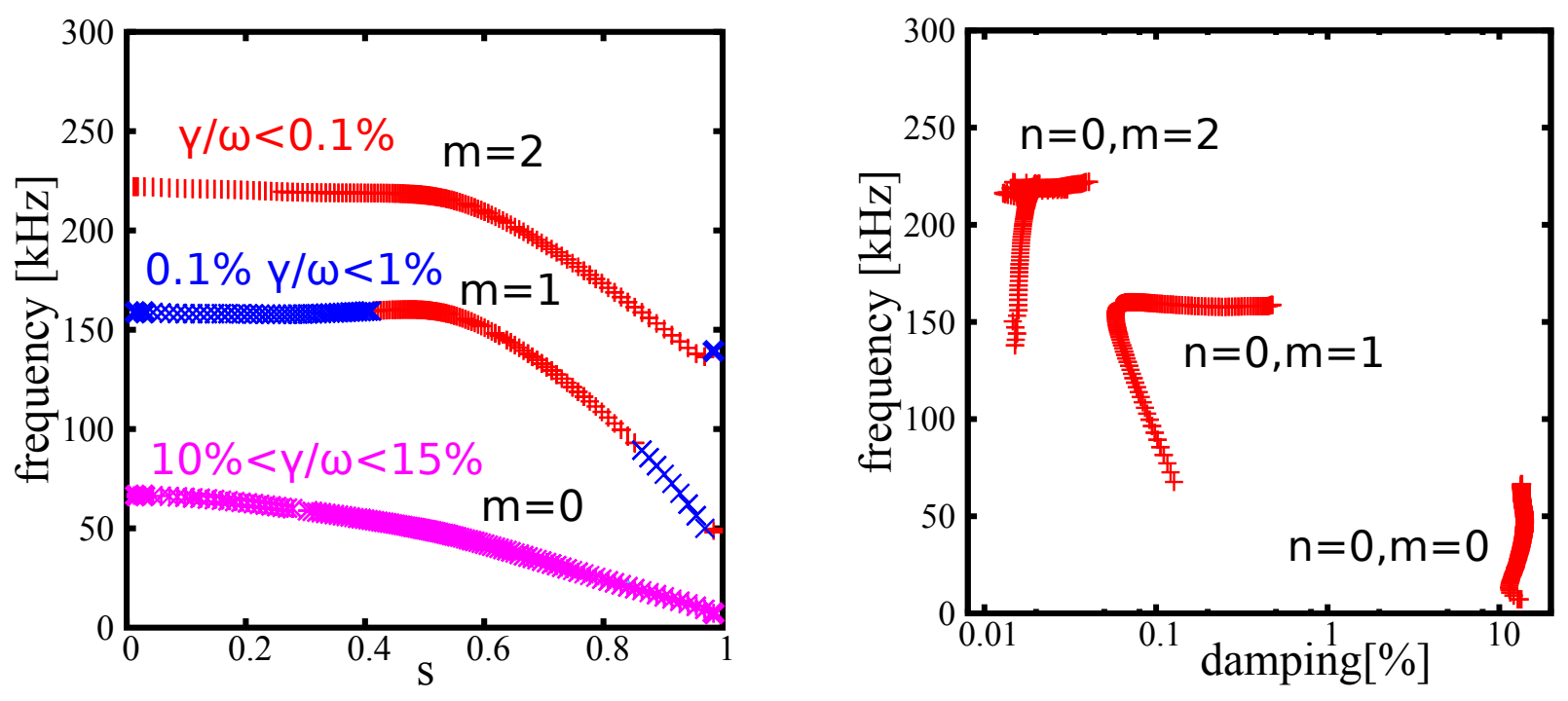

Figure 7. Left: kinetic continuum for $n=0$ : the red crosses show the part of the continuum that has a damping less than $\gamma / \omega<0.1 \%$, the blue diagonal crosses $0.1 \%<\gamma / \omega<1 \%$ and the magenta stars $10 \%<\gamma / \omega<15 \%$; Right: the GAM $(n=0, m=0)$, GAE $(n=0, m=1)$ and the $(n=0, m=2)$ branch (as on the left) in the complex plane. Whereas the damping for the GAM branch is relatively large due to the high background ion temperature, the damping for the 'Alfvénic' branches is very low.
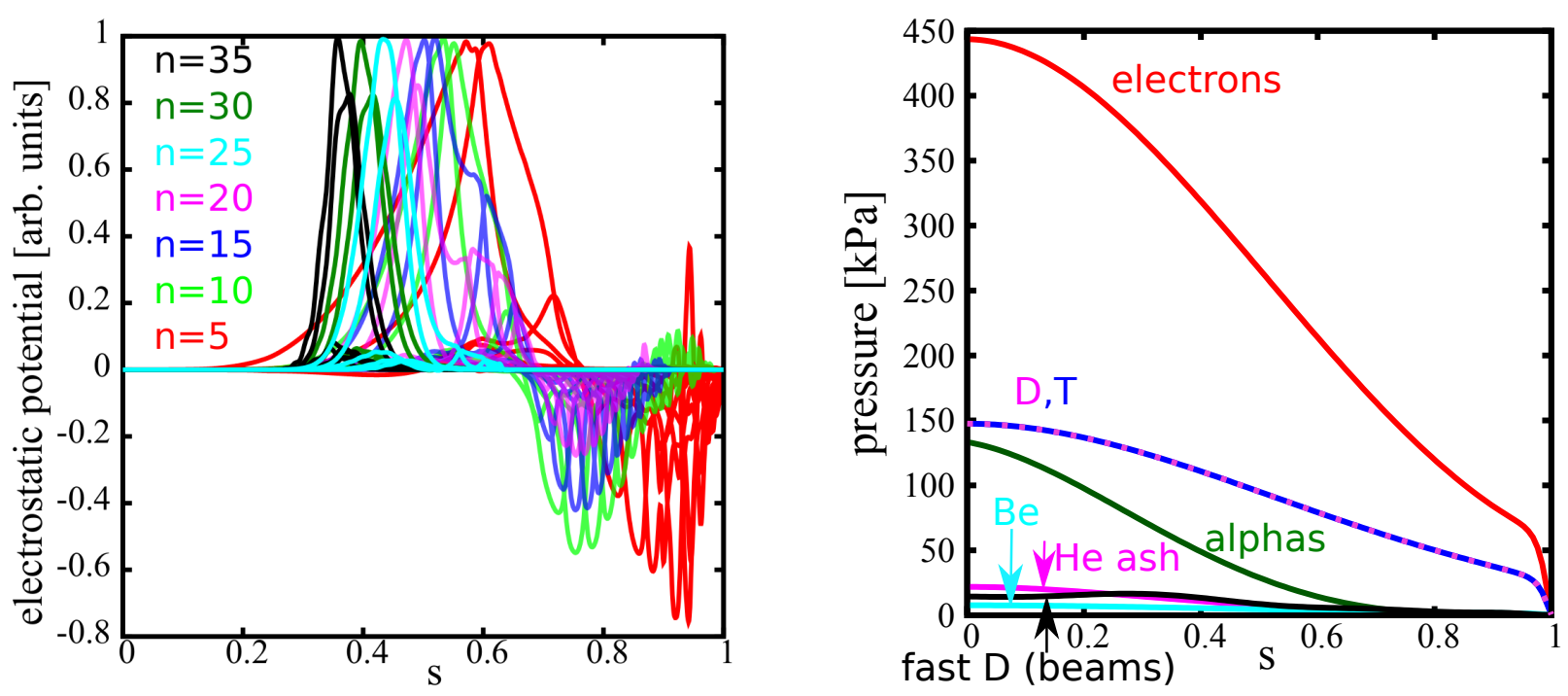

Figure 8. Left: Kinetic even TAE eigenfunctions for $n$ between 5 and 35; Right: plasma pressures due to the different particle species; note the difference between the He ash and the energetic $\alpha$-particles.

increasing mode numbers. For the odd TAE branch, these effects almost compensate each other and therefore only a weak dependence on $n$ was found.

For completeness and benchmarking with other codes also the damping for the EAE (ellipticity induced) gap is given: due to its higher frequency the EAE branch is much less damped (see figure 6). Finally, the $n=0$ branches are discussed briefly: whereas the geodesic acoustic branch is heavily damped (see figure 7), the $m=1$ branch - also called global Alfvén 
eigenmode branch (GAE) - experiences small damping over a large radial range. These branches are not only important because of their role for the interaction with background turbulence [40] but also for benchmarking initial value codes via decay studies of an initial perturbation.

The conclusion of this local analysis chapter is, that all kinetic effects considered here tend to reduce the local damping. Especially the isotope effects and $\omega_{*}$ effects (for higher $n$ ) have a considerable impact.

\subsection{Global analysis}

It has been predicted that the EP transport in ITER can be solved by applying local models [24, 41, 12]. This prediction is based on the fact that the $\alpha$-particle drive is optimal (drift orbit width of driving particles equals the mode width) around $n \sim 30$ for this scenario and on the assumption that the most unstable modes are radially rather localised. More global (and thus more dangerous) modes are thought to be even more damped due to non-local continuum damping [12]. In addition, if the saturation amplitudes are small quasi-linear estimates for the EP transport can be used [24, 42]. However, no non-linear global, multi- $n$ simulations have been carried out to support these predictions. As will be shown in this section, some of these assumptions may only be marginally fulfilled or not be valid at all. Further, it is important to investigate how far the scenarios are from the thresholds that would impact the ITER performance, also in the view of more unstable (advanced) scenarios.

As a first step towards a more complete numerical description, the mode structures, frequencies and damping rates of a large range of toroidal mode numbers are documented here. As a next step [43] this information will be used in non-linear hybrid simulations employing the HAGIS code [44]. Of particular concern for EP transport are rather low$n$ modes $(n<15)$ with a large radial extent and/or dense clusters of modes that cause overlapping resonances in phase space. In both cases EPs can be effectively redistributed [45]. For a final answer on the magnitude of this transport, global, non-linear calculations are necessary: it cannot be ruled out that low- $n$ modes $(n<15)$ are excited in regions of phase space that are not affected by the high- $n$ modes - especially when they are sparse and only affect a small volume in phase space. Also in the non-linear phase the modes with the largest linear growth rate may not always have the largest amplitudes or sub-dominant modes can saturate with the highest amplitude [7, 46] because of non-linear phase space coupling effects.

As described above, for the $q$-profile given in figure 2 the TAE modes form a rather dense cluster of modes (see figure 8). The damping dependencies of these modes can be understood as follows: as shown in figure 9, the mode peak position moves radially outwards with decreasing $n$ (pink squares). At the same time, the mode frequencies decrease, as indicated by the red, green and blue lines. This means that $x=\omega /\left(\left|k_{\|}\right| v_{t h}\right)$ (see section 2) changes via $\omega$ and $v_{t h, i} \sim \sqrt{T_{i}}$. Since neither $\omega$ nor $v_{t h, i}$ change linearly, and $x$ is an argument in $e^{-x^{2}}$, the damping behaviour as shown on the right of figure 9 is rather complex. In addition, radiative damping becomes larger for more localised mode structures via its dependence on 
Local and global kinetic stability analysis of Alfvén eigenmodes in the 15MA ITER scenario11
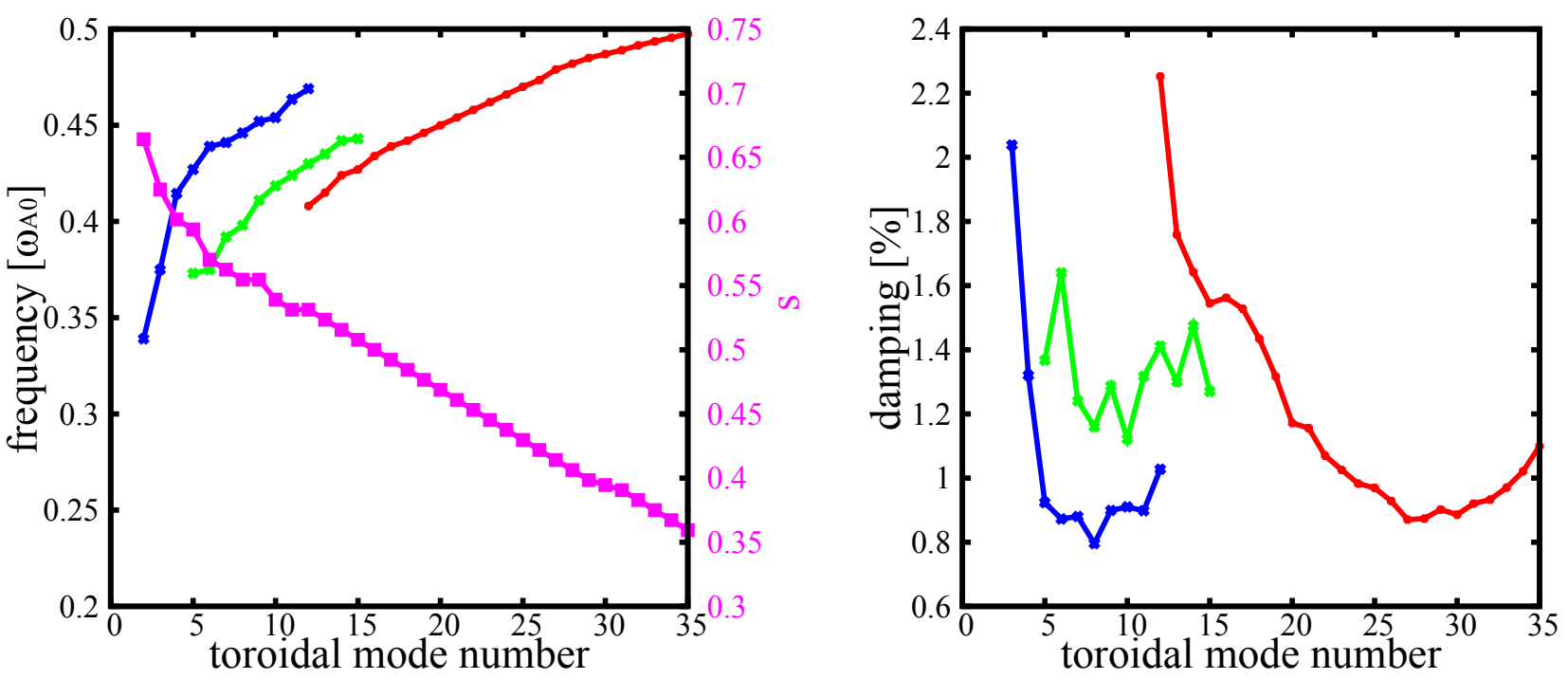

Figure 9. Left: mode frequency and radial position of the TAEs as function of their toroidal mode number $\left(q_{0}=0.99\right)$; Right: damping as a function of the toroidal mode number; the colours in both plots refer to different TAE branches: red for TAEs with the dominant poloidal harmonics $(m, m+1)$; green for $(m+1, m+2)$ blue for $(m+2, m+3)$; the magenta line shows the position of the main TAE gap as a function of $s$.

$k_{\perp} \varrho_{i}$, whereas on the other hand the modes move into the low shear region for higher $n$ 's that in turn decreases radiative damping. Finally, for $n \approx 20$ the modes become very localised and they do not couple through several gaps (see figure 8). Therefore, there is no interaction with the continuum at the plasma edge $(s \approx 0.85)$. For lower mode numbers, continuum damping tends to increase since the distance between the closed edge continuum and the main TAE gap decreases.

Interestingly, at $n \approx 15$ other branches of the TAEs start to appear (see figure 9, green and blue lines). These modes couple in a different way through the outer gaps (see figure 10) than the lowest frequency TAE modes in figure 8. In fact, for $n=10$ and below the dominant poloidal harmonics are not $m_{1}=n, m_{2}=n+1$ but $m_{1}=n+1, m_{2}=n+2$. Surprisingly, the damping of these mode is rather low. On the one hand, due to their radial location around $s \sim 0.6$, they avoid strong ion Landau damping and also the radiative damping is small (low $n$ ). On the other hand, continuum damping is expected to increase for modes with low $n$. In this case, however, the rather strong shear between $s \sim 0.6-0.85$ causes the mode's poloidal harmonics to decrease rather fast while coupling through the outer part of the gap. Therefore, at the intersection with the continuum, the mode's relative amplitude is rather low, and therefore also the continuum damping.

The challenging question for future studies will be, if these type of modes (that are localised outside the region where no strong EP drive is present) could be driven non-linearly unstable even by small EP transport from the more core-localised and (higher $n$ ) modes.

For completeness also an EAE mode is given in figure 6- its damping is low compared to the TAE branches (higher frequency) but also its drive is expected to be considerably smaller that the TAE drive. A dedicated study for EAEs will be carried out elsewhere. 

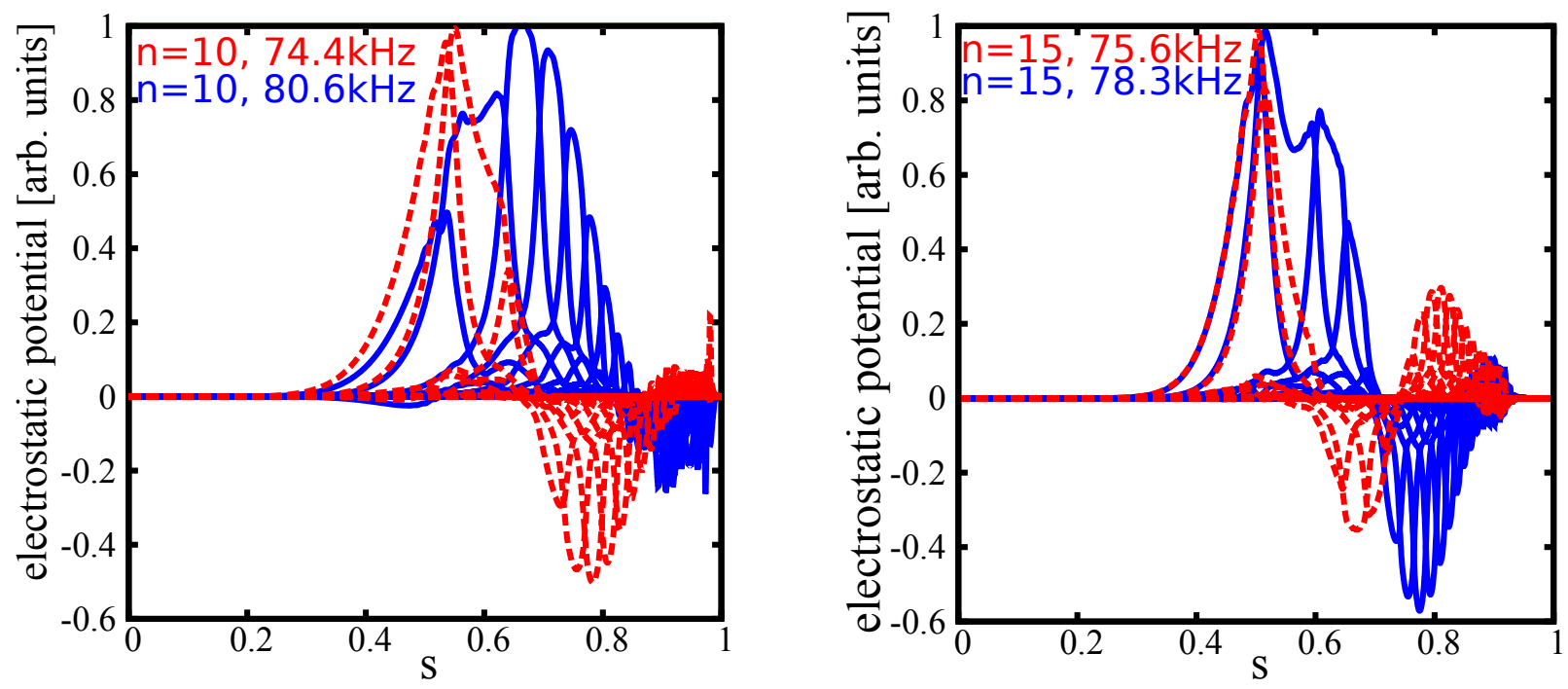

Figure 10. Left: two $n=10$ TAEs at different frequencies belonging to different branches; Right: two $n=15$ TAEs at different frequencies with different coupling through the gap

For the global TAE runs a radial resolution of up to 768 radial grid points with grid refinement close to the continuum intersection point has been used. The number of poloidal harmonics has been between 7 and 33, depending on the nature of the modes: whereas very low- $n$ and very high- $n$ modes require a rather moderate number of poloidal harmonics, the intermediate n's $(n \sim 5 \ldots 15)$ require many harmonics for convergence. Two different solution strategies are used: for high- $n$ modes with a few poloidal harmonics (typically 5 ) an iterative solver can be employed (see chapter 3.2 of ref. [11]), for low- $n$ modes with many harmonics the antenna version of the code is used (chapter 3.3 of ref. [11]). In the high- $n$ limit, both solvers agree perfectly. The run-time for a the largest case is less than 24 hours on 32 cores of a present day Linux cluster. Both the calculation of the matrix elements and the diagonalisation of the matrix are parallelised (WSMP [47]).

\subsection{Energetic particle drive}

Adding energetic $\alpha$-particles according to the density and pressure profiles given in figures 2 and 8 leads to weakly unstable modes. It is beyond the limitations of this paper to include a full study for all toroidal mode numbers with different distribution functions. This work is still in progress and will be reported as soon as possible. However, a few selected cases will be presented here.

Again both solvers, the inverse vector iteration and the antenna version of the code are used. Figure 11 illustrates what happens to the antenna response and phase when the instability threshold is approached: as soon as one crosses the marginal point the phase between the antenna drive and the plasma response jumps. In figure 11 an analytical hot Maxwellian for a TAE with $n=20$ was used. The temperature is chosen to be equivalent to a slowing down distribution function in the sense that $T_{\alpha}=p_{\alpha} / n_{\alpha}$. The numerical calculation (in the same spirit as discussed in figure 1) for the same case leads to a slightly higher threshold (similar 

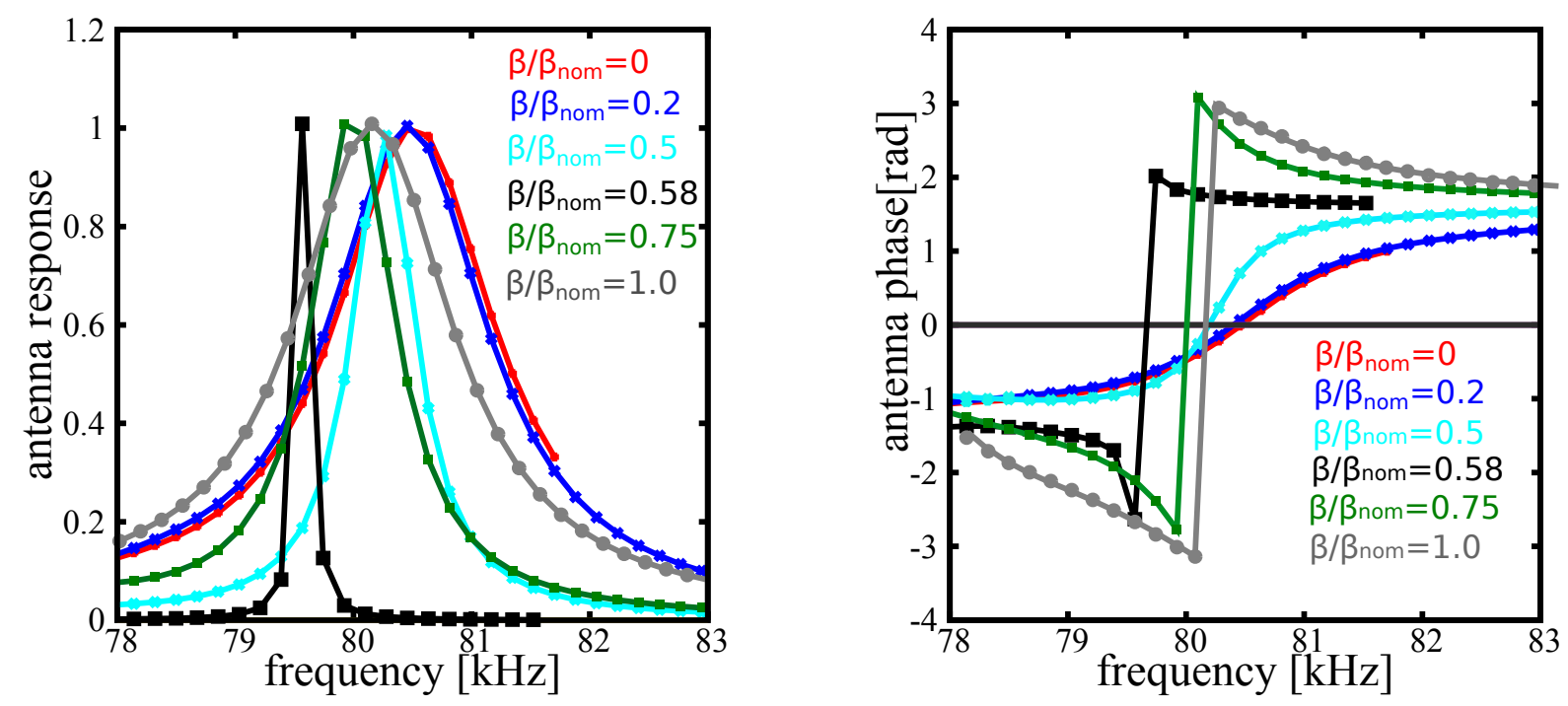

Figure 11. Left: response function ( $n=20 \mathrm{TAE})$ for increasing $\alpha$-particle drive given as the fraction $\beta / \beta_{\text {nom }}$ of the nominal on-axis $\alpha$-particle $\beta_{\text {nom }}=1.15 \%: \beta_{\alpha} / \beta_{\text {nom }}=0$ (red), 0.2 (blue), 0.5 (cyan), 0.58 (black), 0.75 (green) and 1 (grey); Right: phase between antenna drive and mode response shows a jump at the instability threshold.

argumentation as above for ion Landau damping), i.e. $\beta_{\text {crit }}=0.85 \beta_{\text {nom }}$ with $\beta_{\text {nom }}=1.15 \%$. Again the coefficients (as in figure 1) were benchmarked in the appropriate limit. For $n=25$ and $n=30$ the instability threshold lies at $\beta_{\text {crit }}=0.65$, and $\beta_{\text {crit }}=0.6$, respectively. The growth rates at the nominal $\beta_{\text {nom }}$ are $0.31 \%, 1.3 \%$ and $1.5 \%$ for $n=20,25$ and 30 . This is in good agreement with HAGIS calculations for the same case [43], if the background damping is subtracted. The remaining difference of about $0.5 \%$ is probably due to the finite Larmor radius effects of the fast ions that are kept in LIGKA but neglected in HAGIS. Also, the low- $n$ branches are marginally unstable with $\gamma / \omega<0.5 \%$.

The NBI drive is estimated to be of similar importance for the overall drive [36]. Detailed velocity space calculations from slowing down models will be used in a further study to exactly determine its magnitude and to benchmark with other codes.

\section{Conclusions and further work}

Quantitative estimates of the linear instability threshold of AEs in ITER require a fully gyrokinetic treatment for the background species and hot ions. This has been demonstrated in this paper in detail: dilution effects, trapped particles and diamagnetic effects (for medium and high- $n$ cases) have been shown to reduce the linear damping rates (figure 4). In order to include radiative damping consistently, especially in the cases where the TAEs couple through many gaps, a non-perturbative treatment is indispensable. Furthermore, the exact mode structure is crucial to determine the continuum damping (figure 10). Altogether, this illustrates the well known fact that the damping of global TAEs is rather complex since several mechanisms interact in a local and non-local way and thus this work provides a basis for 
benchmarking and for non-linear perturbative studies.

To this end, the damping as a function of the toroidal mode number has been determined (figure 9). For the standard TAE this function has a flat minimum around $n=27$. However, low- $n$ TAEs can have a rather small damping due to their localisation in outer gap regions (figures 9 10]. This result demonstrates that the assumption made in ref.[12] that local TAEs are always less damped than global ones is in general not necessarily fulfilled in this ITER scenario. The $\alpha$-particle drive can destabilise slightly $(\gamma / \omega<1.5 \%)$ the TAEs between $n \sim 20$ and $n \sim 35$. Also, low- $n$ TAEs are marginally unstable.

In summary, this paper tries to document as detailed as possible the importance of kinetic effects on the TAE stability for one ITER reference case. It also indicates that a local treatment may not be sufficient for determining the overall EP transport. The proof to this conjecture, however, has to be left to future non-linear simulations that have been started recently $([43,26])$. The results presented in this paper support the qualitative discussion in the ITER Physics Base, chapter 5 [48, 49], however it adds more detailed, comprehensive and quantitative results concerning the marginal stability threshold for TAEs, that is a very crucial ingredient to all non-linear transport studies.

Clearly, there are several other open issues that have to be addressed in the near future: performing detailed linear EAE and NAE studies, expanding the EP drive to a wider range of toroidal mode numbers, adding the NBI drive and starting non-linear simulations on the basis of these results. Also a sensitivity study concerning the profiles (mainly the densities and the $q$-profiles) is envisaged. As indicated in section 3.2, a $q$-profile with $q_{0}=0.9$ (keeping the shear constant) gives rise to the existence of isolated core localised 'tornado' modes and reduces the density of overlapping TAEs at mid-radius and the outer core. A similar behaviour is expected if $q_{0}$ is raised to $q_{0} \sim 1$. I. If the non-linear picture changes substantially due to these modifications, how 'optimal EP transport' scenarios might look like and how bursty the EP transport will be around the marginal stability profile will be the focus of future investigations.

\section{Acknowledgements}

The author would like to thank in particular S. Sharapov, S.D. Pinches and all the members of the ITPA group on Energetic Particles for motivating this work and sharing analytical results (S. Sharapov). Further, I want to thank A. Polevoi for providing the ITER scenario simulations. Finally, I want to acknowledge fruitful discussions within the Enabling Research Project 'Theory and simulation of energetic particle dynamics and ensuing collective behaviors in fusion plasmas' funded by the Eurofusion Consortium and the MaxPlanck/Princeton Center for Plasma Physics (MPPC). This project has received funding from the European Union Horizon 2020 research and innovation programme under grant agreement number 633053. The views and opinions expressed herein do not necessarily reflect those of the European Commission. 


\section{References}

[1] Ph. Lauber, S. Günter, A. Könies, and S. D. Pinches. LIGKA: A linear gyrokinetic code for the description of background kinetic and fast particle effects on the MHD stability in tokamaks. Journal Of Computational Physics, 226(1):447-465, Sep 2007.

[2] H. Qin, W. M. Tang, and G. Rewoldt. Linear gyrokinetic theory for kinetic magnetohydrodynamic eigenmodes in tokamak plasmas. Physics of Plasmas, 6(6):2544-2562, 1999.

[3] Ph. Lauber, M. Brüdgam, D. Curran, V. Igochine, K. Sassenberg, S. Günter, M. Maraschek, M. Garcia-Munoz, N. Hicks, and the ASDEX Upgrade Team. Kinetic Alfvén eigenmodes at ASDEX Upgrade. Plasma Physics and Controlled Fusion, 51(12):124009, 2009.

[4] W. Deng, Z. Lin, I. Holod, X. Wang, Y. Xiao, and W. Zhang. Gyrokinetic particle simulations of reversed shear Alfvén eigenmode excited by antenna and fast ions. Physics of Plasmas, 17(11):112504, 2010.

[5] H. S. Zhang, Z. Lin, I. Holod, X. Wang, Y. Xiao, and W. L. Zhang. Gyrokinetic particle simulation of beta-induced Alfvén eigenmode. Physics of Plasmas, 17(11):112505, 2010.

[6] H. S. Zhang, Z. Lin, W. Deng, I. Holod, Z. X. Wang, Y. Xiao, and W. L. Zhang. Nonlinear dynamics of beta-induced Alfvén eigenmode in tokamak. Physics of Plasmas, 20(1):012510, 2013.

[7] M. Schneller, Ph. Lauber, M. Brüdgam, S. D. Pinches, and S. Günter. Double-resonant fast particle-wave interaction. Nuclear Fusion, 52(10):103019, 2012.

[8] Y. Chen, T. Munsat, S. E. Parker, W. W. Heidbrink, M. A. Van Zeeland, B. J. Tobias, and C. W. Domier. Gyrokinetic simulations of reverse shear Alfvén eigenmodes in DIII-D plasmas. Physics of Plasmas, 20(1):012109, 2013.

[9] E. M. Bass and R. E. Waltz. Gyrokinetic simulations of mesoscale energetic particle-driven Alfvénic turbulent transport embedded in microturbulence. Physics of Plasmas, 17(11), 2010.

[10] E. M. Bass and R. E. Waltz. Gyrokinetic simulation of global and local Alfvén eigenmodes driven by energetic particles in a DIII-D discharge. Physics of Plasmas (1994-present), 20(1):012508, 2013.

[11] Philipp Lauber. Super-thermal particles in hot plasmas: kinetic models, numerical solution strategies, and comparison to tokamak experiments. Physics Reports, 533(2):33 - 68, 2013.

[12] R.E. Waltz and E.M. Bass. Prediction of the fusion alpha density profile in ITER from local marginal stability to Alfvén eigenmodes. Nuclear Fusion, 54(10):104006, 2014.

[13] $\mathrm{Ph}$. Lauber et al. The influence of plasma shaping effects on the damping of toroidal Alfvén eigenmodes. Proc. 23rd Int. Conf. on Fusion Energy 2010, pages THW/P7-08, 2010.

[14] A. Fasoli, D. Testa, T. Panis, A. Klein, J. A. Snipes, J. Sears, M. Gryaznevich, R. Martin, and S. D. Pinches. Active excitation and damping rate measurement of intermediate-n toroidal Alfvén eigenmodes in JET, C-Mod and MAST plasmas. Plasma Physics and Controlled Fusion, 52(7), 2010.

[15] H. Park, E. Mazzucato, T. Munsat, C. W. Domier, M. Johnson, Jr. N. C. Luhmann, J. Wang, Z. Xia, I. G. J. Classen, A. J. H. Donné, and M. J. van de Pol. Simultaneous microwave imaging system for density and temperature fluctuation measurements on TEXTOR. Review of Scientific Instruments, 75(10):3787-3792, 2004.

[16] B. J. Tobias. Imaging techniques for microwave diagnostics. Contributions to plasma physics, 51(2-3):111, 2011.

[17] B. J. Tobias, I. G. J. Classen, C. W. Domier, W. W. Heidbrink, N. C. Luhmann, R. Nazikian, H. K. Park, D. A. Spong, and M. A. Van Zeeland. Fast ion induced shearing of 2D Alfvén eigenmodes measured by Electron Cyclotron Emission Imaging. Phys. Rev. Lett., 106:075003, Feb 2011.

[18] I. G. J. Classen, J. E. Boom, W. Suttrop, E. Schmid, B. Tobias, C. W. Domier, Jr. N. C. Luhmann, A. J. H. Donné, R. J. E. Jaspers, P. C. de Vries, H. K. Park, T. Munsat, M. García-Mu noz, and P. A. Schneider. 2D Electron Cyclotron Emission Imaging at ASDEX Upgrade. Review of Scientific Instruments, 81(10):10D929, 2010.

[19] M. Garcia-Munoz, H. . U. Fahrbach, and H. Zohm. Scintillator based detector for fast-ion losses induced by magnetohydrodynamic instabilities in the ASDEX Upgrade tokamak. Review of Scientific Instruments, 80(5):053503, May 2009.

[20] W. W. Heidbrink, N. N. Gorelenkov, Y. Luo, M. A. Van Zeeland, R. B. White, M. E. Austin, K. H. Burrell, G. J. Kramer, M. A. Makowski, G. R. McKee, and R. Nazikian. Anomalous flattening of the fast-ion profile during Alfvén-Eigenmode activity. Phys. Rev. Lett., 99:245002, Dec 2007.

[21] B. Geiger, M. Garcia-Munoz, W. W. Heidbrink, R. M. McDermott, G. Tardini, R. Dux, R. Fischer, V. Igochine, and the ASDEX Upgrade Team. Fast-ion d-alpha measurements at ASDEX Upgrade. Plasma Physics and Controlled Fusion, 53(6):065010, 2011.

[22] N. N. Gorelenkov et al. The linear stability properties of medium- to high-n TAEs in ITER. PPPL Report 4287, 2008.

[23] C.Z. Cheng. Kinetic extensions of magnetohydrodynamics for axisymmetric toroidal plasmas. Physics Reports, 211(1):1 - 51, 1992.

[24] Ron Waltz and Eric Bass. Prediction of the fusion alpha density profile in iter from local marginal stability to Alfvén eigenmodes. 13th IAEA Technical Meeting on Energetic Particles in Magnetic Confinement Systems (1720 September 2013), 2013.

[25] R. R. Mett and S. M. Mahajan. Kinetic theory of toroidicity-induced Alfvén eigenmodes. Physics of Fluids B: Plasma Physics, 4(9):2885-2893, 1992

[26] M. Schneller et al. Study of Nonlinear Fast Particle Transport in the Presence of Alfvén Waves for the ITER 15 MA Scenario. Proc. 25th Int. Conf. on Fusion Energy 2014, pages TH/P2-06, 2014.

[27] G. Hogeweij et al. Impact of W on Scenario Simulations for ITER. Proc. 25th Int. Conf. on Fusion Energy 2014, pages EX/P3-17, 2014.

[28] F. Zonca, L. Chen, and R.A. Santoro. Kinetic theory of low-frequency Alfvén modes in tokamaks. Plasma Physics and Controlled Fusion, 38(11):2011-2028, 1996. 
[29] F. Zonca, L. Chen, A. Botrugno, P. Buratti, A. Cardinali, R. Cesario, and V.P. Ridolfini. High-frequency fishbones at JET: theoretical interpretation of experimental observations. Nuclear Fusion, 49(8):085009, Aug 2009.

[30] G. Y. Fu and J. W. Van Dam. Excitation of the toroidicity-induced shear Alfvén eigenmode by fusion alpha particles in an ignited tokamak. Physics of Fluids B: Plasma Physics, 1(10):1949-1952, 1989.

[31] J. Candy and M. N. Rosenbluth. Nonideal theory of toroidal Alfvén eigenmodes. Physics of Plasmas (1994-present), 1(2):356-372, 1994.

[32] Z. Gao, L. Peng, P. Wang, J. Dong, and H. Sanuki. Plasma elongation effects on temperature gradient driven instabilities and geodesic acoustic modes. Nuclear Fusion, 49(4):045014, 2009.

[33] I. Chavdarovski and F. Zonca. Effects of trapped particle dynamics on the structures of a low-frequency shear Alfvén continuous spectrum. Plasma Physics and Controlled Fusion, 51(11):115001, 2009.

[34] Ph. Lauber, I.G.J. Classen, D. Curran, V. Igochine, B. Geiger, S. da Graca, M. Garcia-Munoz, M.Maraschek, P. McCarthy, and the ASDEX Upgrade Team. NBI-driven Alfvénic modes at ASDEX Upgrade. Nuclear Fusion, 52(9):094007, 2012.

[35] A.R. POLEVOI, S.Y. MEDVEDEV, V.S. MUKHOVATOV, A.S. KUKUSHKIN, Y. MURAKAMI, M. SHIMADA, and A.A. IVANOV. ITER Confinement and Stability Modelling. Journal of Fusion Research, 5:82-97, 2002.

[36] S. D. Pinches et al. Energetic ions in iter plasmas. in preparation (2014), 2014.

[37] M Saigusa, H Kimura, Y Kusama, G J Kramer, T Ozeki, S Moriyama, T Oikawa, Y Neyatani, and T Kondoh. Systematic study of toroidicity-induced Alfvén eigenmodes at low- q discharges in JT-60U. Plasma Physics and Controlled Fusion, 40(9):1647, 1998.

[38] G.J. Kramer, C.Z. Cheng, Y. Kusama, R. Nazikian, S. Takeji, and K. Tobita. Magnetic safety factor profile before and after sawtooth crashes investigated with toroidicity and ellipticity induced Alfvén eigenmodes. Nuclear Fusion, 41(9):1135, 2001.

[39] T. Gassner, K. Schoepf, S. E. Sharapov, V. G. Kiptily, S. D. Pinches, C. Hellesen, and J. Eriksson. Deuterium beam acceleration with 3rd harmonic ion cyclotron resonance heating in Joint European Torus: Sawtooth stabilization and Alfvén eigenmodes. Physics of Plasmas, 19(3):032115, Mar 2012.

[40] K.G. McClements, L.C. Appel, M.J. Hole, and A. Thyagaraja. Excitation of axisymmetric Alfvénic modes in ohmic tokamak discharges. Nuclear Fusion, 42(9):1155, 2002.

[41] W.W. Heidbrink, M.A. Van Zeeland, M.E. Austin, E.M. Bass, K. Ghantous, N.N. Gorelenkov, B.A. Grierson, D.A. Spong, and B.J. Tobias. The effect of the fast-ion profile on Alfvén eigenmode stability. Nuclear Fusion, 53(9):093006, 2013.

[42] K. Ghantous, N. N. Gorelenkov, H. L. Berk, W. W. Heidbrink, and M. A. Van Zeeland. 1.5D quasilinear model and its application on beams interacting with Alfvén eigenmodes in DIII-D. Physics of Plasmas, 19(9):092511, Sep 2012.

[43] M. Schneller et al. Study of nonlinear fast particle transport and losses in the presence of Alfvén waves. THEORY OF FUSION PLASMAS: JOINT VARENNA - LAUSANNE INTERNATIONAL WORKSHOP Villa Monastero, Varenna, Italy; September 15, 2014, 2014.

[44] S. D. Pinches, L. C. Appel, J. Candy, S. E. Sharapov, H. L. Berk, D. Borba, B. N. Breizman, T. C. Hender, K. I. Hopcraft, G. T. A. Huysmans, and W. Kerner. The HAGIS self-consistent nonlinear wave-particle interaction model. Computer Physics Communications, 111(1-3):133-149, 1998.

[45] H.L. Berk, B.N. Breizman, J. Fitzpatrick, and H.V. Wong. Line broadened quasi-linear burst model [fusion plasma]. Nuclear Fusion, 35(12):1661, 1995.

[46] S.D. Pinches. Nonlinear Interaction of Fast Particles with Alfvén waves in Tokamaks. Ph.D. Thesis, The University of Nottingham, 1996.

[47] Anshul Gupta and Mahesh Joshi. WSMP: A high-performance shared- and distributed-memory parallel sparse linear equation solver. 2001.

[48] ITER Physics Expert Group on Energetic Particles Heating and Current Drive et al. Energetic particle behaviour. Nuclear Fusion, 39:2471, 1999.

[49] K. Ikeda et a. Progress in the ITER physics basis. Nucl. Fusion, 47(6), 2007. 